\title{
Analysis and Simulation of 2ASK Digital Band-pass Transmission System Basing on SIMULINK
}

\author{
Geng Wang ${ }^{1, a^{*}}$ and Wei Cheng ${ }^{1, b}$ \\ ${ }^{1}$ The School of Electronic and Information Engineering, Hubei University of Science and \\ Technology, Xianning, 437100, China \\ awanggeng20001982@126.com, b799040609@qq.com
}

\section{Keywords: SIMULINK; 2ASK; Modulation; Demodulation; BER}

\begin{abstract}
Digital modulation and demodulation system as an important part of digital communication system, its research is necessary. Based on the analysis of 2ASK digital band-pass system transmission characteristics, SIMULINK software is used to simulate the digital band-pass transmission system. Having been fully considered the possible factors affecting the 2ASK signal in the transmission process, a basic model of 2ASK digital band-pass transmission system is established, a system model which can simulate and realize the function of 2ASK digital band-pass signal transmission. Debugging and testing show that the waveforms of the system are consistent with the theoretical analysis and error-free code transmission can be achieved; the symbol error rate is rising with the increase of noise, which indicates that the system design is effective and reasonable. It is worth reference for related research and the improvement of such system, and also provides a new way for system design and experimental teaching.
\end{abstract}

\section{Introduction}

The digital communication system has the advantages of strong anti-interference ability, controllable transmission error, easy to transmit and exchange, easy to store, easy to integrate, easy to be encrypted and so on. It has incomparable advantage of analog communication and is widely used. Because the digital baseband signal has a rich low-frequency components, it is difficult to effectively carry out wireless transmission or long-distance cable transmission, you must first digital baseband signal carrier modulation, the spectrum suitable for channel transmission, then the receiver will transform the Signal to restore. This process of converting the spectrum of the digital baseband signal is called digital modulation, and the process of restoring the received signal to the digital baseband signal at the receiving end is called digital demodulation[1]. In the digital communication system, commonly used digital modulation and demodulation techniques are amplitude shift keying (ASK), frequency shift keying (FSK), phase shift keying PSK) [2-4].

The design and analysis of the communication system is a complicated project, and the rapid development of computer simulation technology makes it possible to replace the traditional hardware simulation communication experiment system. There are many computer languages, such as MATLAB/SIMULINK[5], SystemView[6], LabVIEW[7] and so can be used for communication system simulation, which, Simulink is provided by Matlab for dynamic system modeling, simulation and analysis toolkit. Simulink provides a module specifically for displaying the output signal, which can be viewed at any time during the simulation. Simulink memory module can be used to save simulation data to Matlab workspace or file in various ways, so that users can analyze and process the data after the end of simulation. In addition, Simulink has a specific function of the code organized into modules, and these modules can be organized into a hierarchical structure of the subsystem, so the inherent modular design. Based on the above advantages, Simulink as a universal simulation modeling tool, widely used in communication simulation, digital signal processing, fuzzy logic, digital control neural network, mechanical control and virtual reality and other fields. In this paper, MATLAB/SIMULINK 2ASK communication system for simulation and design. 


\section{ASK Modulation and Demodulation Principle}

Digital signal transmission is divided into baseband transmission and band-pass transmission, in practice, most of the channel has a band-pass characteristics can not be directly transmitted baseband signal. In order for the digital signal to be transmitted in the bandpass channel, the digital baseband signal must be used to modulate the carrier to match the characteristics of the channel to the channel. This digital baseband signal with the carrier control, the digital baseband signal is converted to digital band-pass signal is called digital modulation process. Digital modulation is typically performed using keying, such as the amplitude, frequency, and phase of the carrier[8]. Amplitude-shift keying is a digital modulation of the amplitude of the carrier as the digital baseband signal changes, and its frequency and initial phase remain constant. When the modulation signal is a binary digital signal, this modulation is called binary digital modulation. In the 2ASK modulation, the amplitude of the carrier only two changes in the state, that is, the use of digital information " 0 " or " 1 " baseband rectangular pulse to a continuous carrier wave, the carrier intermittent output. "1" is output when a carrier wave is output, and "0" is transmitted when no carrier wave is output. 2 ASK signal can be expressed as :

$e_{0}(t)=s(t) \cos \omega_{c} t$

Where A is the carrier angular frequency and $\mathrm{s}(\mathrm{t})$ is the unipolar NRZ rectangular pulse train.

$$
s(t)=\sum_{a} a_{n} g\left(t-n T_{b}\right)
$$

Where $\mathrm{s}(\mathrm{t})$ is a rectangular pulse of duration $\mathrm{T}_{\mathrm{b}}$ and height 1 , and is often called a gate function; 2ASK signal waveform shown in Fig. 1:

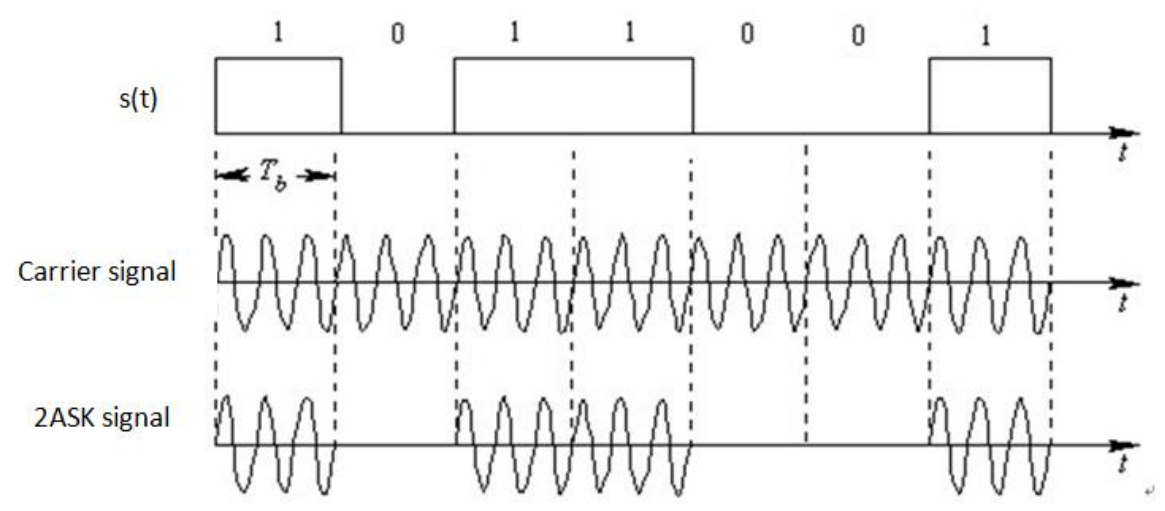

Figure 1. 2ASK signal waveform

There are two ways to realize ASK modulation: 1. Multiplier implementation method 2. Key control method. Multiplier implementation of the input method is a random sequence of information, through the baseband signal generator, resulting in a waveform sequence, the multiplier used for spectrum shift, the multiplication of the signal through the band-pass filter to filter out high-frequency harmonics and low-frequency interference. The keying method is another method of generating the ASK signal. Binary ASK is also known as on-off control (OOK) [9]. The most typical implementation is achieved by using a key to control the output of the carrier oscillator[10].

ASK demodulation in two ways: 1. envelope detection method 2. Coherent demodulation. Synchronous demodulation, also known as coherent demodulation[11], the signal through the band-pass filter to suppress out-of-band interference from the channel, the multiplier to reverse the removal of the spectrum to restore the baseband signal[12]. The low-pass filter is used to suppress high-order harmonics generated by the multiplier. Since the envelope of the AM signal waveform is proportional to the input baseband signal $\mathrm{m}(\mathrm{t})$, it is also possible to recover the original modulated signal by envelope detection. Envelope detector generally consists of half-wave or full-wave rectifier and low-pass filter. Coherent detection method block diagram shown in Fig. 2. Coherent detection is synchronous mediation, requiring the receiver to generate a carrier wave with the carrier frequency in phase with the local carrier signal, called synchronous carrier or coherent carrier. This carrier is multiplied with the received modulated signal and the output is: 


$$
z(t)=y(t) \cos \omega_{c} t=s(t) \cos ^{2} \omega_{c} t=\frac{1}{2} s(t)+\left(1+\cos 2 \omega_{c} t\right)
$$

After the second high-frequency component is filtered out by the low-pass filter, the $s(t)$ signal is output. The cut-off frequency of the low-pass filter is equal to the highest frequency of the baseband digital signal. Because of the influence of noise and transmission characteristics, low-pass filter output waveform distortion, the sampling decision, after shaping digital baseband pulse regeneration.

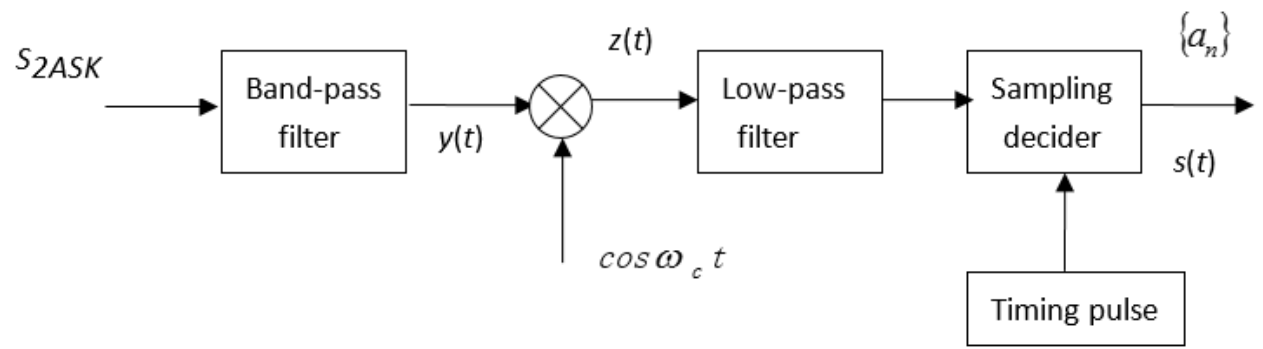

Figure 2. 2ASK Coherent Demodulation

\section{ASK Digital Band-pass Transmission System Simulation}

The modulation and demodulation process of the ASK simulation system is as follows: the output signal of the signal source and the carrier are multiplied by the multiplier and transmitted into the additive white Gaussian noise (AWGN) channel. The receiver through the band-pass filter and then multiplied by the carrier, and then through the low-pass filter, sampling judge, and finally by the oscilloscope shows the various stages of waveform, and the error rate observed with the error code. In the MATLAB simulink simulation platform to build the ASK modulation and demodulation simulation circuit shown in Fig. 3.

In a modem system, the carrier signal frequency is generally greater than the frequency of the signal source. The signal source frequency is $1 \mathrm{~Hz}$, so the carrier frequency is set to $6 \mathrm{~Hz}$, since in the carrier parameter setting, the unit of the frequency is $\mathrm{rad} / \mathrm{sec}$, so it is $12 *$ pi. The lower frequency of the bandpass filter should equal the difference between the carrier frequency and the modulation signal frequency. The upper frequency should be equal to the sum of the carrier frequency and the modulation signal frequency. In front of the signal source has been set to $1 \mathrm{~Hz}$ frequency, carrier frequency is $6 \mathrm{~Hz}$, calculated for the upper and lower cut-off frequency of $7 \mathrm{~Hz}, 5 \mathrm{~Hz}$, converted to rads / sec for the unit is $14 *$ pi, $10 *$ pi. Set the parameters, the simulation, the output waveform from the oscilloscope shows that the signal modulation and demodulation is successful, but there is a delay of 0.01 seconds, that is, the signal delay of 2 bits (with the delay time sampling multiplied by the sampling frequency of the sampling encoder ).

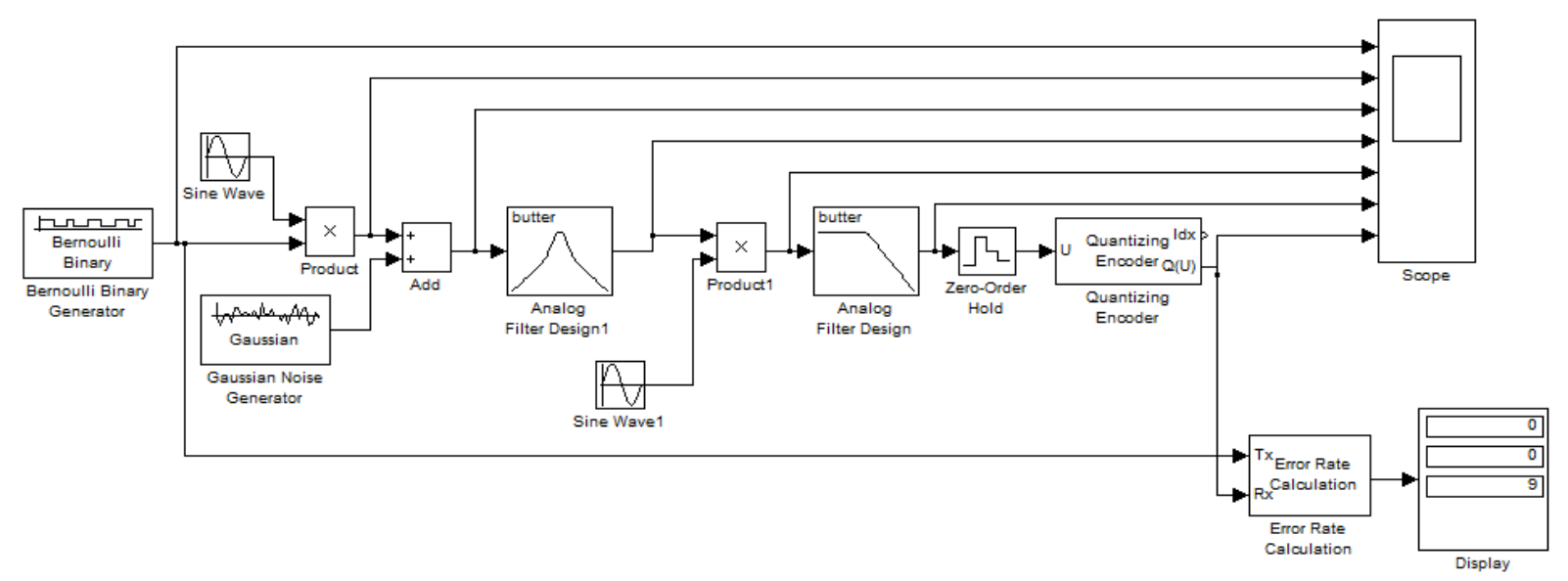

Figure 3. 2ASK Simulation Test Model 


\section{Simulation Results and Analysis}

After the input signal through the ASK modem system, the output of each waveform (from top to bottom are the input signal, carrier signal, modulated signal, through the multiplier demodulation signal, through the low-pass filter demodulation signal output Signal) the first channel for the signal source module waveform, the second for the ASK modulated waveform, the third channel to join the white Gaussian noise after the waveform, the fourth pass through the band-pass filter after the waveform, The fifth channel is the waveform after multiplying the carrier by the band-pass filter, the sixth is the waveform after the low-pass filter, and the seventh is the waveform after ASK demodulation. In the ASK modulation and demodulation by adding white Gaussian noise, the waveform distortion, there are errors in demodulation, the system basically meet the design requirements. As shown in Fig. 4.

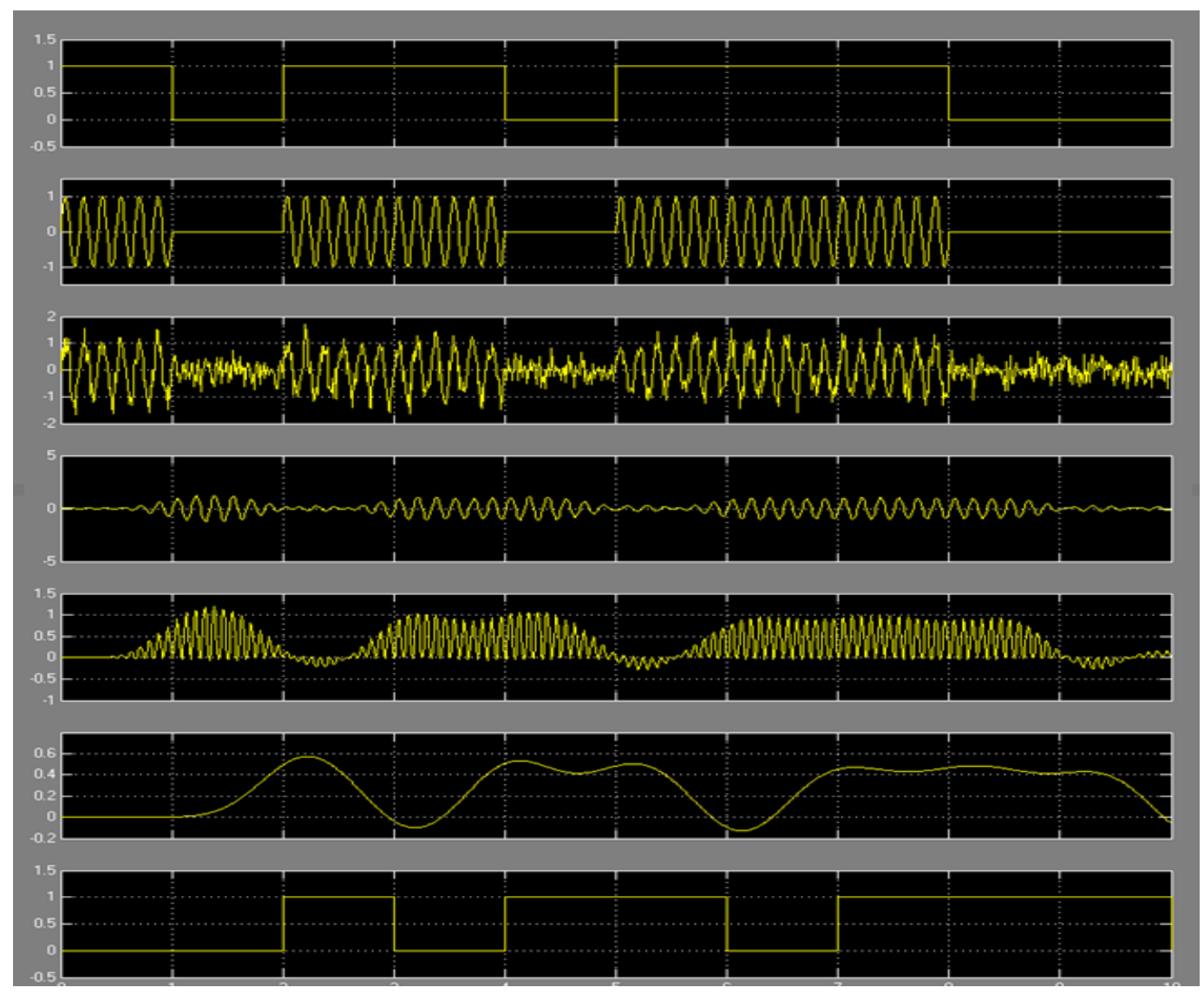

Figure 4. The Waveform of Each Point Signal

\section{Conclusion}

This paper completes the simulation design of 2ASK transmission system based on MATLAB / Simulink. By adjusting the experimental parameters and comparing the experimental results, we can get or verify the required or existing conclusions in a vivid way so that the experimental results can be more clear, so that students can better understand the basic concepts and principles of communication systems. MATLAB / Simulink to a number of complex procedures become very intuitive, easy to operate, and easy to modify and later maintenance, can be used as a teacher classroom teaching auxiliary teaching software, the abstract concept of concrete, visualization, to deepen understanding, memory, To improve the effect of teaching quality, so as to mobilize the enthusiasm of students and improve students' innovative ability, with practical significance. 


\section{Acknowledgment}

This work was supported by the Hubei University of Science and Technology Teaching Research Project(2015-XB-007).

\section{References}

[1] Alshahrie A, Mahmoud S W Z. Multimode modeling of digital modulation in nearly single-mode semiconductor lasers[J]. Physics of Wave Phenomena, 2016, 24(2): 114-123.

[2] Xiang $\mathrm{P}$, Zheng $\mathrm{X}$, Zhang $\mathrm{H}$, et al. A novel approach to photonic generation of RF binary digital modulation signals[J]. Optics express, 2013, 21(1): 631-639.

[3] Li W, Li M, Zhu N H. All-optical generation of ASK, PSK, and FSK radio-frequency signals[C]//Progress in Electromagnetic Research Symposium (PIERS). IEEE, 2016: 2362-2362.

[4] Abuelma'atti M T, Khalifa Z J. A new memristor emulator and its application in digital modulation[J]. Analog Integrated Circuits and Signal Processing, 2014, 80(3): 577-584.

[5] Kiyan M, Bingöl E, Melikoğlu M, et al. Modelling and simulation of a hybrid solar heating system for greenhouse applications using Matlab/Simulink[J]. Energy Conversion and Management, 2013, 72: 147-155.

[6] LI L, WU D. Simulation and Design of AM Communication System Based on the System View Software[J]. Research and Exploration in Laboratory, 2015, 9: 024.

[7] Alfuth E J, Chester T, Roisen N, et al. A Diy Langmuir trough Made with Adruino, LabVIEW, and 3D Printed Parts for Education and Research[J]. Biophysical Journal, 2015, 108(2): 333a.

[8] Gómez J T, Hernández F, Habermann J. New digital demodulator with matched filters andcurve segmentation techniques for BFSK demodulation: FPGA implementation and results[J]. Ingeniería e Investigación, 2016, 36(1): 105-110.

[9] Kim H S, Kim J K, Park K B, et al. On/Off control of boost PFC converters to improve light-load efficiency in paralleled power supply units for servers[J]. IEEE Transactions on Industrial Electronics, 2014, 61(3): 1235-1242.

[10] Hong C H, Wu C Y, Liao Y T. Robustness enhancement of a Class-C quadrature oscillator using capacitive source degeneration coupling [J]. IEEE Transactions on Circuits and Systems II: Express Briefs, 2015, 62(1): 16-20.

[11] Gao F, Zheng Y, Feng X, et al. Thermoacoustic resonance effect and circuit modelling of biological tissue[J]. Applied Physics Letters, 2013, 102(6): 063702.

[12] Dörfler F, Chertkov M, Bullo F. Synchronization in complex oscillator networks and smart grids[J]. Proceedings of the National Academy of Sciences, 2013, 110(6): 2005-2010. 